\title{
Finite Speed of the Perturbation Distribution and Asymptotic Behavior of the Solutions of a Parabolic System not in Divergence Form
}

\author{
Alisher Matyakubov \\ Department of Applied Mathematics and Computer Analysis, National University of Uzbekistan, Uzbekistan
}

Copyright $\subset 2017$ by authors, all rights reserved. Authors agree that this article remains permanently open access under the terms of the Creative Commons Attribution License 4.0 International License

\begin{abstract}
The property of a finite speed of a perturbation distribution to the Cauchy problem for a parabolic system not in divergence form based on comparison method and an asymptotic behavior of a self-similar solution for both slow and fast diffusion cases are established. It is shown that the coefficients of the main term of the asymptotic of solution satisfy some system of nonlinear algebraic equations. It is found the Zeldovich-Kompaneets-Barenblatt type solution to the parabolic system.
\end{abstract}

Keywords Not in Divergence Form, Finite Speed, Perturbation, Global Solutions, Asymptotic Behavior, Numerical Analysis

\section{Introduction}

In this paper we consider the parabolic system of equations not in divergence form with Cauchy conditions

$$
\begin{aligned}
& \frac{\partial u}{\partial t}=v^{m_{1}} \frac{\partial}{\partial x}\left(\left|\frac{\partial u}{\partial x}\right|^{p-2} \frac{\partial u}{\partial x}\right), \\
& \frac{\partial v}{\partial t}=u^{m_{2}} \frac{\partial}{\partial x}\left(\left|\frac{\partial v}{\partial x}\right|^{p-2} \frac{\partial v}{\partial x}\right),(t, x) \in Q \\
& u(0, x)=u_{0}(x), v(0, x)=v_{0}(x), x \in R
\end{aligned}
$$

where $\mathrm{Q}=\{(\mathrm{t}, \mathrm{x}): \mathrm{t}>0, \mathrm{x} \in \mathrm{R}\}, \mathrm{p}, \mathrm{m}_{1}, \mathrm{~m}_{2}$ are the numerical parameters, $u=u(t, x) \geq 0, v=v(t, x) \geq 0$ are the solutions.

Not in divergence form equations and system of equations are often used to describe various physical phenomena, such as the diffusive process for biological species, the resistive diffusion phenomena in force-free magnetic fields, curve shortening flow, spreading of infectious disease and so on, see for [1-15].

The equations and system of equations not in divergence form, comparing with the classical divergence form equations are more close to the actual circumstances in some cases. For example, for the biological species, the diffusion of divergence form implies that the species is able to move to all locations within its environment with equal probability, but if we consider this problem with the objective conditions, the population density will affects the rate of diffusion, so a kind of 'biased' diffusion equation will be more realistic, for the non-divergence form diffusion. The diffusion rate is regulated by population density that is increasing for large populations and decreasing for small populations.

It is observed by many authors [1-15, 19-21] nonlinear equations of non-divergence form are a source of new nonlinear effects, such as finite speed of a perturbation of distribution, space localization, blow up, etc. In particular, nonlinear problems in the non-divergence form of the following form

$$
\begin{aligned}
& u_{t}=u^{p}(\Delta u+a u), \mathrm{x} \in \Omega, \mathrm{t}>0, \\
& \mathrm{u}(x, 0)=u_{0}(x), \mathrm{x} \in \Omega \\
& u(x, t)=0, \mathrm{x} \in \partial \Omega, \mathrm{t}>0
\end{aligned}
$$

are discussed by several authors, see Friedman and Mcleod [1], Gage [2], Passo and Luckhaus [3], Wang [4] et al.

Let $\lambda_{1}$ be the first eigenvalue of $\Delta$ in $\Omega$ with homogeneous Dirichlet boundary condition. In paper [1], Friedman and Mcleod proved the existence of global solutions if $\lambda_{1}>\mathrm{a}$ and $\mathrm{p}=2$ in (3), and the solution has a blow-up property if $\lambda_{1}<\mathrm{a}$ and $\mathrm{p}=2$. In [2] Gage studied in more detail the solutions with the blow-up property for $\mathrm{p}=2$ and set the estimates for the blow-up solution. In [5] for the cases $\lambda_{1}>\mathrm{a}$ and $\mathrm{p}>2$ in problem (3), Zimmer studied the existence of global solutions. In paper [6], Chen studied the solution of problem (1) with the blow-up property 
in the domain $\Omega$ for $\lambda_{1}<\mathrm{a}, 1<\mathrm{p}<2$ and large initial data. In [7], Wiegner considered problem (3) for the case $p>1$ and proved that the solution of problem (3) has the blow-up property for $\lambda_{1}<\mathrm{a}$. In [4], Wang et al. studied problem (3) for $p>1$. They obtained uniqueness conditions for the solution and proved that all positive solutions of problem (3) exist globally if and only if $\lambda_{1} \geq a$. In paper [3], Passo and Luckhaus studied some properties of solutions of problem (3).

Later, some authors generalized the problem (3). In [8], Raimbekov investigated some properties of solutions of the Cauchy problem for a nonlinear degenerate parabolic equation in a non-divergence form with variable density $|x|^{n} \frac{\partial u}{\partial t}=u^{m} \operatorname{div}\left(|\nabla u|^{p-2} \nabla u\right)$. He studied a self-similar

solution of the Zeldovich-Kompaneets-Barenblatt type, established the asymptotics of self-similar solutions in the case of fast and slow diffusion.

In [9], Zhou et al. investigated positive solutions of a doubly degenerate parabolic equation in a non-divergent form with a gradient term

$$
\frac{\partial u}{\partial t}=u^{m} \operatorname{div}\left(|\nabla u|^{p-2} \nabla u\right)+\lambda u^{q}+\gamma u^{r}|\nabla u|^{p}
$$

with zero Dirichlet boundary conditions. They established the local existence of weak solutions of the problem, and then determined how the gradient term affects the behavior of the solutions.

Nonlinear systems of equations in a non-divergence form were discussed in the papers of Wang, Xie et al.

In [10] Wang and Xie considered for the system $u_{t}=v^{p}(\Delta u+a v), \quad v_{t}=u^{q}(\Delta v+b u)$ with null Dirichlet boundary conditions and positive initial conditions, where $\mathrm{p}, \mathrm{q}, \mathrm{a}$ and $\mathrm{b}$ are all positive constants. The authors proved that: (i) when $\min \{a, b\} \leq \lambda_{1}$, where $\lambda_{1}$ is the first eigenvalue of $\Delta$ in $\Omega$ with homogeneous Dirichlet boundary condition, then there exists global positive classical solution, and all positive classical solutions cannot blow up in finite time in the meaning of maximum norm; (ii) when $\min \{a, b\}>\lambda_{1}$, there is no global positive classical solutions.

In [11], Wang studied for the system $u_{t}=u^{p}(\Delta u+a v)$, $v_{t}=v^{q}(\Delta v+b u)$ with null Dirichlet boundary conditions and positive initial conditions, where $\mathrm{p}, \mathrm{q}, \mathrm{a}$ and $\mathrm{b}$ are all positive constants. He proved that all positive classical solutions of problem exist globally, if and only if $\lambda_{1}^{2} \geq a b$.

In [12] $\mathrm{H}$. Lu deals with positive solutions of some degenerate and quasilinear parabolic systems not in divergence form

$$
\begin{aligned}
& u_{i t}=f_{i}\left(u_{i+1}\right)\left(\Delta u_{i}+a_{i} u_{i}\right), x \in \Omega, t>0, i=1,2, \ldots, n-1, \\
& u_{n t}=f_{n}\left(u_{1}\right)\left(\Delta u_{n}+a_{n} u_{n}\right), x \in \Omega, t>0 .
\end{aligned}
$$

On some restrictions to the functions $f_{i}\left(u_{i+1}\right), i=1,2, \ldots, n-1$, he proved the local existence and uniqueness of classical solution. Moreover, it was proven that: if $\min \left\{a_{1}, \ldots, a_{n}\right\} \leq \lambda_{1}$ there exists global positive classical solution, and all positive classical solutions cannot blow up in finite time in the meaning of maximum norm. Also, in this reference it is shown that if $\min \left\{a_{1}, \ldots, a_{n}\right\}>\lambda_{1}$, and the initial datum $\left(u_{10}, \ldots, u_{n 0}\right)$ satisfies to some assumptions, then the positive classical solution is unique and blows up in finite time.

Later, in [19-21], the authors studied the Cauchy problem for the system

$$
\begin{aligned}
& |x|^{n} \frac{\partial u}{\partial t}=v^{m_{1}} \nabla\left(|x|^{k} u^{m_{1}-1} \nabla u\right)+|x|^{n}{ }_{\mathrm{u}}^{\beta_{1}}, \\
& |x|^{n} \frac{\partial v}{\partial t}=u^{m_{2}} \nabla\left(|x|^{k} v^{m_{2}-1} \nabla v\right)+|x|^{n} v^{\beta_{2} .} .
\end{aligned}
$$

They studied the properties of self-similar solutions of the system in a two-component medium with variable density and source, in particular, solutions of Zeldovich-Kompaneets-Barenblatt type for cross-diffusion systems of a non-divergent type were constructed, and slow and fast diffusion cases were investigated.

Finite speed perturbation of distribution (FSPD) property and asymptotic behavior of self-similar solutions for divergence systems are considered in [15-18].

The system (1) is degenerate. Therefore it does not have classical solution in the domain $\mathrm{Q}$ at $u=0, v=0, \frac{\partial u}{\partial x}=0$, or $\frac{\partial v}{\partial x}=0$. In this case we consider weak solution with assumptions $u(t, x) \geq 0, v(t, x) \geq 0$ and we introduce the following definitions of solution (see $[8,10,11,13,14]$ ).

Definition 1 (weak solution). A nonnegative functions $u(t, x), v(t, x)$ defined in $Q$ are called a weak solution of the Cauchy problem (1)-(2) with $\left|\frac{\partial u}{\partial x}\right|^{p-2} \frac{\partial u}{\partial x} \in C(Q)$, $\left|\frac{\partial v}{\partial x}\right|^{p-2} \frac{\partial v}{\partial x} \in C(Q), \quad$ if $\quad u(t, x) \geq 0, v(t, x) \geq 0$, $\lim _{t \rightarrow t_{0}} \int_{\Omega} u(t, x) \eta(x) d x=\int_{\Omega} u\left(t_{0}, x\right) \eta(x) d x \quad$ for $\quad$ any $\eta(x) \in C_{0}^{1}(Q)$ and $(u, v)$ satisfies the system (1) in the sense of the integral identity.

Definition 2 (compactly supported solution). A solution $u(t, x), v(t, x)$ of system (1) at each $t \in(0,+\infty)$ is finite with respect to $x$, if the following conditions $u(t, x) \equiv 0$, 
$v(t, x) \equiv 0, \quad x \geq l(t) \quad$ and $\quad u(t, x)>0, \quad v(t, x)>0 \quad$ at $x \leq l(t), t \in(0,+\infty)$ valid.

Definition 3 (vanishing-at-infinity solution). A solution $u(t, x), v(t, x)$ of system (1) determined on the domain $Q$ is called vanishing-at-infinity if $u(t, x) \rightarrow 0, \quad v(t, x) \rightarrow 0$ for $t \rightarrow+\infty$.

In this paper we study asymptotic behavior of self-similar solutions for a degenerate parabolic system not in divergence form (1) for slow and the fast diffusion cases. And based on comparison method the property FSPD of the Cauchy problem for a cross-diffusion parabolic system not in divergence form is established. On the basis of this asymptotic of solutions, suitable initial approximations are offered in the iterative process for the slow and fast diffusion cases, depending on the values of the numeric parameters.

\section{The Self-similar System of Equations}

Here we provide a method of nonlinear splitting [14] for construction of self-similar system of equations. We look for the solutions $u(t, x), v(t, x)$ in the form

$$
\begin{aligned}
& \mathrm{u}(\mathrm{t}, \mathrm{x})=(\mathrm{T}+\mathrm{t})^{-\alpha_{1}} \mathrm{w}(\tau(\mathrm{t}), \mathrm{x}), \\
& \mathrm{v}(\mathrm{t}, \mathrm{x})=(\mathrm{T}+\mathrm{t})^{-\alpha_{2}} \psi(\tau(\mathrm{t}), \mathrm{x}),
\end{aligned}
$$

where

$$
\begin{aligned}
& \tau(\mathrm{t})=\left\{\begin{array}{l}
\frac{(\mathrm{T}+\mathrm{t})^{\sigma}}{\sigma}, \text { at } \sigma>0, \\
-\frac{(\mathrm{T}+\mathrm{t})^{\sigma}}{\sigma}, \text { at } \sigma<0, \\
\ln (\mathrm{T}+\mathrm{t}), \text { at } \sigma=0,
\end{array}\right. \\
& \sigma=1-\alpha_{1}(\mathrm{p}-2)-\alpha_{2} \mathrm{~m}_{1}, \mathrm{~T}>0 .
\end{aligned}
$$

It is easy to check that for unknown functions $w, \psi$ we obtain the following system of equations

$$
\begin{aligned}
& \frac{\partial \mathrm{w}}{\partial \tau}=\psi^{\mathrm{m}_{1}} \frac{\partial}{\partial \mathrm{x}}\left(\left|\frac{\partial \mathrm{w}}{\partial \mathrm{x}}\right|^{\mathrm{p}-2} \frac{\partial \mathrm{w}}{\partial \mathrm{x}}\right)+\mathrm{b}_{1} \tau^{-1} \mathrm{w}, \\
& \frac{\partial \psi}{\partial \tau}=\mathrm{w}^{\mathrm{m}_{2}} \frac{\partial}{\partial \mathrm{x}}\left(\left|\frac{\partial \psi}{\partial \mathrm{x}}\right|^{\mathrm{p}-2} \frac{\partial \psi}{\partial \mathrm{x}}\right)+\mathrm{b}_{2} \tau^{-1} \psi,
\end{aligned}
$$

if $\alpha_{1}(p-2)+\alpha_{2} m_{1}=\alpha_{2}(p-2)+\alpha_{1} m_{2}, \quad 1-\alpha_{1}(p-2)-\alpha_{2} m_{1} \neq 0$, where $b_{i}=\frac{\alpha_{i}}{1-\alpha_{i}(p-2)-\alpha_{3-i} m_{i}}, i=1,2$ and in the case $1-\alpha_{1}(\mathrm{p}-2)-\alpha_{2} \mathrm{~m}_{1}=0 \quad$ we have a system

$$
\begin{aligned}
& \frac{\partial \mathrm{w}}{\partial \tau}=\psi^{\mathrm{m}_{1}} \frac{\partial}{\partial \mathrm{x}}\left(\left|\frac{\partial \mathrm{w}}{\partial \mathrm{x}}\right|^{\mathrm{p}-2} \frac{\partial \mathrm{w}}{\partial \mathrm{x}}\right)+\alpha_{1} \mathrm{w}, \\
& \frac{\partial \psi}{\partial \tau}=\mathrm{w}^{\mathrm{m}} \frac{\partial}{\partial \mathrm{x}}\left(\left|\frac{\partial \psi}{\partial \mathrm{x}}\right|^{\mathrm{p}-2} \frac{\partial \psi}{\partial \mathrm{x}}\right)+\alpha_{2} \psi .
\end{aligned}
$$

Put in (5) and (6)

$$
\begin{aligned}
& \mathrm{w}(\tau, \mathrm{r})=\mathrm{f}(\xi), \psi(\tau, \mathrm{r})=\phi(\xi), \\
& \xi=\left\{\begin{array}{l}
\mathrm{x} \tau^{-1 / p}, \text { at } 1-\alpha_{1}(\mathrm{p}-2)-\alpha_{2} \mathrm{~m}_{1} \neq 0, \\
\mathrm{c} \tau-\mathrm{x}, \text { at } 1-\alpha_{1}(\mathrm{p}-2)-\alpha_{2} \mathrm{~m}_{1}=0,
\end{array}\right.
\end{aligned}
$$

where $\xi$ is the self-similar variable.

If $1-\alpha_{1}(p-2)-\alpha_{2} m_{1}>0$ we obtain the self-similar system of equations

$$
\begin{aligned}
& \phi^{\mathrm{m}_{1}} \frac{\mathrm{d}}{\mathrm{d} \xi}\left(\left|\frac{\mathrm{df}}{\mathrm{d} \xi}\right|^{\mathrm{p}-2} \frac{\mathrm{df}}{\mathrm{d} \xi}\right)+\frac{\xi}{\mathrm{p}} \frac{\mathrm{df}}{\mathrm{d} \xi}+\mathrm{b}_{1} \mathrm{f}=0, \\
& \mathrm{f}^{\mathrm{m}_{2}} \frac{\mathrm{d}}{\mathrm{d} \xi}\left(\left|\frac{\mathrm{d} \phi}{\mathrm{d} \xi}\right|^{\mathrm{p}-2} \frac{\mathrm{d} \phi}{\mathrm{d} \xi}\right)+\frac{\xi}{\mathrm{p}} \frac{\mathrm{d} \phi}{\mathrm{d} \xi}+\mathrm{b}_{2} \phi=0 .
\end{aligned}
$$

And in the case $\alpha_{i}=\frac{p-2-m_{i}}{(p-2)^{2}-m_{1} m_{2}}, i=1,2$ we have a self-similar system

$$
\begin{aligned}
& \phi^{m_{1}} \frac{d}{d \xi}\left(\left|\frac{d f}{d \xi}\right|^{p-2} \frac{d f}{d \xi}\right)-c \frac{d f}{d \xi}+\alpha_{1} f=0, \\
& f^{m_{2}} \frac{d}{d \xi}\left(\left|\frac{d \phi}{d \xi}\right|^{p-2} \frac{d \phi}{d \xi}\right)-c \frac{d \phi}{d \xi}+\alpha_{2} \phi=0 .
\end{aligned}
$$

It is easy to check that following functions $\mathrm{u}(\mathrm{t}, \mathrm{x})=(\mathrm{T}+\mathrm{t})^{-\alpha_{1}} \overline{\mathrm{f}}(\xi), \mathrm{v}(\mathrm{t}, \mathrm{x})=(\mathrm{T}+\mathrm{t})^{-\alpha_{2}} \bar{\phi}(\xi), \quad(10) \xi=\mathrm{x} / \tau^{1 / \mathrm{p}}$, $\tau(\mathrm{t})=\frac{(\mathrm{T}+\mathrm{t})^{1-\alpha_{1}(\mathrm{p}-2)-\alpha_{2} \mathrm{~m}_{1}}}{1-\alpha_{1}(\mathrm{p}-2)-\alpha_{2} \mathrm{~m}_{1}}, \quad 1-\alpha_{1}(\mathrm{p}-2)-\alpha_{2} \mathrm{~m}_{1}>0$ are the Zeldovich-Kompaneets-Barenblatt type solution of the system (1) if numbers $\alpha_{i}, i=1,2$ satisfy the conditions $\alpha_{1}(p-2)+\alpha_{2} m_{1}=\alpha_{2}(p-2)+\alpha_{1} m_{2}, \frac{\alpha_{i}}{1-\alpha_{i}(p-2)-\alpha_{3-i} m_{i}}=\frac{1}{p}, i=1,2$.

In accordance with the statement of the original problem we will consider nontrivial, nonnegative solutions of the system equations (8)-(9) satisfying the following conditions:

$$
\begin{aligned}
& \mathrm{f}(0)=\mathrm{M}_{1}, \phi(0)=\mathrm{M}_{2}, \mathrm{M}_{1}^{\mathrm{p}-2-\mathrm{m}_{2}}=\mathrm{M}_{2}^{\mathrm{p}-2-\mathrm{m}_{1}}, \\
& \mathrm{f}\left(\mathrm{d}_{1}\right)=\phi\left(\mathrm{d}_{2}\right)=0,0<\mathrm{d}_{1}<\infty, 0<\mathrm{d}_{2}<\infty .
\end{aligned}
$$

\section{Slow Diffusion (Case $p-2>m$,}

$$
\left.\mathrm{m}=\max \left\{\mathrm{m}_{1}, \mathrm{~m}_{2}\right\}\right)
$$


Applying the method of comparison of solutions [13] and method of standard equations [14] for solving the problem (8), (10) we obtain the estimates for the solution of the problem (1)-(2).

It should be noted, that the functions

$$
\begin{aligned}
& \bar{f}(\xi)=\left\{\begin{array}{l}
A\left(a-\xi^{\gamma}\right)_{+}^{\gamma_{1}}, \text { at } 1-\alpha_{1}(p-2)-\alpha_{2} m_{1} \neq 0, \\
M_{1} \exp (-\xi), \text { at } 1-\alpha_{1}(p-2)-\alpha_{2} m_{1}=0,
\end{array}\right. \\
& \bar{\phi}(\xi)=\left\{\begin{array}{l}
B\left(a-\xi^{\gamma}\right)_{+}^{\gamma 2}, \text { at } 1-\alpha_{1}(p-2)-\alpha_{2} m_{1} \neq 0, \\
M_{2} \exp (-\xi), \text { at } 1-\alpha_{1}(p-2)-\alpha_{2} m_{1}=0,
\end{array}\right.
\end{aligned}
$$

where

$$
\gamma=\frac{\mathrm{p}}{\mathrm{p}-1}, \gamma_{\mathrm{i}}=\frac{(\mathrm{p}-1)\left(\mathrm{p}-2-\mathrm{m}_{\mathrm{i}}\right)}{(\mathrm{p}-2)^{2}-\mathrm{m}_{1} \mathrm{~m}_{2}}, \mathrm{i}=1,2,
$$

(b) $+\max (0, b)$,

$\mathrm{Aa}^{\gamma_{1}}=\mathrm{M}_{1}, \quad \mathrm{Ba}^{\gamma_{2}}=\mathrm{M}_{2} \quad$ at $\quad \xi<\mathrm{a}(\mathrm{p}-1) / \mathrm{p} \quad$ satisfy the conditions (10).

Theorem 1. Let $\mathrm{p}-2>\mathrm{m}, \mathrm{m}=\max \left\{\mathrm{m}_{1}, \mathrm{~m}_{2}\right\}$,

$$
\begin{gathered}
-\frac{1}{p}+b_{i} \leq 0, \quad A^{p-2} B^{m_{1}} \gamma\left(\gamma_{1}-m_{1} \gamma_{2}\right)\left|\gamma \gamma_{1}\right|^{p-2}=\frac{1}{p}, \\
\left.B^{p-2} A^{m_{2} \gamma\left(\gamma_{2}-m_{2} \gamma_{1}\right) \mid \gamma \gamma_{2}}\right|^{p-2}=\frac{1}{p}, \\
u_{+}(0, x) \geq u_{0}(x), \quad v_{+}(0, x) \geq v_{0}(x), x \in R .
\end{gathered}
$$

Then the classical solution (u, v) of the problem (1)-(2) exists globally and it satisfies estimation

$$
\begin{aligned}
& \mathrm{u}(\mathrm{t}, \mathrm{x}) \leq \mathrm{u}_{+}(\mathrm{t}, \mathrm{x})=(\mathrm{T}+\mathrm{t})^{-\alpha_{1}} \overline{\mathrm{f}}(\xi), \\
& \mathrm{v}(\mathrm{t}, \mathrm{x}) \leq \mathrm{v}_{+}(\mathrm{t}, \mathrm{x})=(\mathrm{T}+\mathrm{t})^{-\alpha_{2}} \bar{\phi}(\xi),
\end{aligned}
$$

in $\mathrm{Q}$.

Proof. Theorem 1 is proved by comparing solution methods. As comparable functions we take the functions $\mathrm{u}_{+}(\mathrm{t}, \mathrm{x}), \mathrm{v}_{+}(\mathrm{t}, \mathrm{x})$ defined by (12). Then, according (8) and

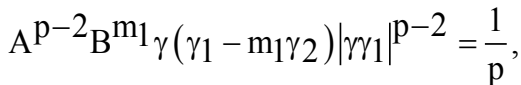

$$
\begin{aligned}
& \left.{ }_{\mathrm{B}}^{\mathrm{p}-2} \mathrm{~A}^{\mathrm{m}}{ }_{2} \gamma_{\left(\gamma_{2}-\mathrm{m}_{2} \gamma_{1}\right) \mid \gamma \gamma_{2}}\right|^{\mathrm{p}-2}=\frac{1}{\mathrm{p}},
\end{aligned}
$$

we obtain

$$
\begin{aligned}
& \mathrm{Lu}_{+}(\mathrm{t}, \mathrm{x})=\left(-\frac{1}{\mathrm{p}}+\mathrm{b}_{1}\right) \overline{\mathrm{f}}(\xi), \\
& \mathrm{Lv}_{+}(\mathrm{t}, \mathrm{x})=\left(-\frac{1}{\mathrm{p}}+\mathrm{b}_{2}\right) \bar{\phi}(\xi) .
\end{aligned}
$$

From these expressions, we find, that for execution, the inequalities $\mathrm{Lu}_{+} \leq 0, \mathrm{Lv}_{+} \leq 0$ are enough to fulfill the conditions $-\frac{1}{p}+b_{i} \leq 0, i=1,2$. From the stated condition in the theorem, the inequalities are satisfied. Then, by the comparison theorem of solutions there is a global solution in $\mathrm{Q}$ for the problem (1)-(2) with the following estimates

$$
u_{+}(t, x) \geq u(t, x), \quad v_{+}(t, x) \geq v(t, x), x \in R
$$

The proof of the theorem 1 is completed.

Thus, we obtained FSPD property of the solutions of the Cauchy problem (1), (2).

\section{The Asymptotic Behavior of Self-similar Solutions of the Problem (8), (9), (11)}

We introduce the notations:

$$
\begin{aligned}
& \mathrm{b}_{1 \mathrm{i}}=\mathrm{p}^{-\mathrm{p}}\left(\gamma_{\mathrm{i}}-1\right)^{-1}\left|\frac{\mathrm{p}-1}{\gamma_{\mathrm{i}}}\right|^{\mathrm{p}-2}, \\
& \mathrm{~b}_{2 \mathrm{i}}=\mathrm{ca}(\mathrm{p}-1) / \mathrm{p}^{1-\mathrm{p}}\left(1-\gamma_{\mathrm{i}}\right)^{-1}\left|\alpha_{\mathrm{i}}\right|^{2-\mathrm{p}}, \quad \mathrm{i}=1,2 .
\end{aligned}
$$

Assume $\alpha_{1}(p-2)+\alpha_{2} m_{1}=\alpha_{2}(p-2)+\alpha_{1} m_{2}$. Then the following theorems are valid.

Theorem 2. Let $1-\alpha_{1}(p-2)-\alpha_{2} m_{1} \neq 0, \quad m_{i}<1, i=1,2$. Then compactly supported solution of the problem (8), (11) has an asymptotic

$$
f(\xi)=c_{1}\left(a-\xi^{\frac{p}{p-1}}\right)^{\gamma_{1}}, \phi(\xi)=c_{2}\left(a-\xi^{\frac{p}{p-1}}\right)^{\gamma_{2}},
$$

at $\xi_{\xi \rightarrow a} \frac{p-1}{p}$, where the coefficients $c_{i}, i=1,2$ are solution of the systems of nonlinear algebraic equations

$$
c_{i}^{p-2} c_{3-i} m_{i}=b_{1 i}, i=1,2 .
$$

Theorem 3. Let $1-\alpha_{1}(p-2)-\alpha_{2} m_{1}=0$, $\mathrm{m}_{\mathrm{i}}>1, \mathrm{i}=1,2$. Then compactly supported solution of the problem (9), (11) at $\xi \rightarrow a^{(p-1) / p}$ has the asymptotic form (14), where the coefficients $c_{i}, i=1,2$ are solution of the systems of nonlinear algebraic equations

$$
c_{i}^{p-2 ~} c_{3-i}^{m}=b_{2 i}, i=1,2 .
$$

Proof. We transform the system (8)-(9) into the relatively easy form. For such auxiliary systems of equations we apply the following transformations 


$$
\begin{aligned}
& \mathrm{f}(\xi)=\overline{\mathrm{f}}(\xi) \mathrm{y}_{1}(\eta), \phi(\xi)=\bar{\phi}(\xi) \mathrm{y}_{2}(\eta), \\
& \eta=-\ln \left(\mathrm{a}-\xi^{\mathrm{p} /(\mathrm{p}-1)}\right)
\end{aligned}
$$

where $\bar{f}(\xi)=c_{1}\left(a-\xi^{\frac{p}{p-1}}\right)^{\gamma_{1}}, \bar{\phi}(\xi)=c_{2}\left(a-\xi^{\frac{p}{p-1}}\right)^{\gamma_{2}}$,

$\mathrm{y}_{1}(\eta), \mathrm{y}_{2}(\eta)$ are unknown functions.

Now let us investigate the asymptotic behavior of

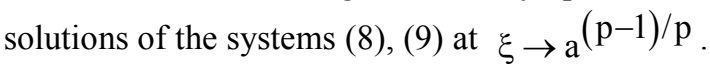

After the transformation (15) the systems (8), (9) will take the following form

$$
\begin{aligned}
& y_{3-i}^{m_{i}} \frac{d}{d \eta}\left(L y_{i}\right)++a_{i 1}(\eta) y_{3-i}^{m_{i}} L_{i}++a_{i 3}(\eta) y_{i}+ \\
& +a_{i 2}(\eta)\left(\frac{d y_{i}}{d \eta}+a_{i 0}(\eta) y_{i}\right)=0, i=1,2 . \\
& \text { Here } L y_{i}=\left|\frac{d y_{i}}{d \eta}+a_{i 0}(\eta) y_{i}\right| p-2\left(\frac{d y_{i}}{d \eta}+a_{i 0}(\eta) y_{i}\right) \text {, } \\
& \mathrm{a}_{\mathrm{i} 0}(\eta)=-\frac{(\mathrm{p}-1)\left(\mathrm{p}-2-\mathrm{m}_{\mathrm{i}}\right)}{(\mathrm{p}-2)^{2}-\mathrm{m}_{1} \mathrm{~m}_{2}}, \\
& a_{i 1}(\eta)=(p-1)\left(\frac{e^{-\eta}}{p\left(a-e^{-\eta}\right)}-\gamma_{i}+1\right), \\
& a_{i 2}(\eta)=\left\{\begin{array}{l}
\frac{(p-1)^{p-1}}{p^{p}}, \text { at } 1-\alpha_{1}(p-2)-\alpha_{2} m_{1} \neq 0, \\
-c \frac{(p-1)^{p-1}}{p^{p-1}}\left(a-e^{-\eta}\right)^{\frac{p-1}{p}}, \text { otherwise, }
\end{array}\right. \\
& a_{i 3}(\eta)=\left\{\begin{array}{l}
\left(\frac{p-1}{p}\right)^{p} \frac{b_{i} e^{-\eta}}{a-e^{-\eta}}, \text { at } 1-\alpha_{1}(p-2)-\alpha_{2} m_{1} \neq 0, \\
\left(\frac{p-1}{p}\right)^{p} \frac{\alpha_{i} e^{-\eta}}{a-e^{-\eta}}, \text { at } 1-\alpha_{1}(p-2)-\alpha_{2} m_{1}=0 .
\end{array}\right.
\end{aligned}
$$

$\mathrm{i}=1,2$.

There was supposed that $\xi \in\left[\xi_{0}, \xi_{1}\right), 0<\xi_{0}<\xi_{1}$,

$$
\xi_{1}=a^{\frac{p}{p-1}} \text {. }
$$

Therefore, the function $\eta(\xi)$ has the properties: $\eta^{\prime}(\xi)>0$ at $\xi \in\left[\xi_{0}, \xi_{1}\right), \eta_{0}=\eta\left(\xi_{0}\right)>0, \lim _{\xi \rightarrow \xi_{1}} \eta(\xi)=+\infty$.

It follows from self-similar system of equations (16) at the following limitations

$$
\lim _{\eta \rightarrow+\infty} a_{i j}(\eta)=a_{i j}^{0} \quad(i=1,2 ; j=0,1,2,3)
$$

are exist, and finite and nonzero, that is $0<\left|\mathrm{a}_{\mathrm{ij}}^{0}\right|<+\infty$.

Further, we consider the solutions of the system (16), in a certain neighborhood $+\infty$ satisfies the inequalities

$$
y_{i}(\eta)>0, \quad y_{i}^{\prime}+a_{i 0}(\eta) y_{i} \neq 0 \quad(i=1,2) .
$$

Assuming in the system (16)

$$
v_{i}(\eta)=\left.\left|\frac{d y_{i}}{d \eta}+a_{i 0}(\eta) y_{i}\right|\right|^{p-2}\left(\frac{d y_{i}}{d \eta}+a_{i 0}(\eta) y_{i}\right),
$$

we obtain the identity

$$
\begin{aligned}
& v_{i}^{\prime}(\eta) \equiv-a_{i 1}(\eta) v_{i}(\eta)-a_{i 2}(\eta) v_{i}^{\frac{1}{p-1}}(\eta) y_{3-i}-m_{i} \\
& -a_{i 3}(\eta) y_{i} y_{3-i}-m_{i}-, \quad(i=1,2) .
\end{aligned}
$$

Consider now the functions

$$
\begin{aligned}
& g_{i}\left(\lambda_{i}, \eta\right) \equiv-a_{i 1}(\eta) \lambda_{i}-a_{i 2}(\eta) \lambda_{i}^{\frac{1}{p-1}} y_{3-i}-m_{i}- \\
& -a_{i 3}(\eta) y_{i} y_{3-i}-m_{i},
\end{aligned}
$$

where $\lambda_{i} \in \mathrm{R}, \quad(\mathrm{i}=1,2)$.

Suppose first $1-\alpha_{1}(p-2)-\alpha_{2} m_{1} \neq 0$.

$$
\begin{aligned}
& \text { Then } \lim _{\eta \rightarrow+\infty} a_{i 2}(\eta)=\frac{(p-1)^{p-1}}{p^{p}}, \\
& \lim _{\eta \rightarrow+\infty} a_{i 1}(\eta)=(p-1)\left(1-\gamma_{i}\right), \quad \lim _{\eta \rightarrow+\infty} a_{i 3}(\eta)=0,(i=1,2)
\end{aligned}
$$

and the functions $g_{i}\left(\lambda_{i}, \eta\right) \quad(i=1,2)$ preserves sign on some interval $\left[\eta_{1},+\infty\right) \subset\left[\eta_{0},+\infty\right)$ for every fixed value $\lambda_{i}$ $(\mathrm{i}=1,2)$.

Now let $1-\alpha_{1}(p-2)-\alpha_{2} m_{1}=0$. It is easy to see that

$$
\lim _{\eta \rightarrow+\infty} a_{i 1}(\eta)=(p-1)\left(1-\gamma_{i}\right), \quad \lim _{\eta \rightarrow+\infty} a_{i 3}(\eta)=0,
$$

$\lim _{\eta \rightarrow+\infty} a_{i 2}(\eta)=-\frac{c(p-1)^{p-1} a^{\frac{p-1}{p}}}{p^{p}}, \quad(i=1,2) \quad$ and the

functions $g_{i}\left(\lambda_{i}, \eta\right) \quad(i=1,2)$ for every fixed value $\lambda_{i}(i=1,2)$ preserves sign on some interval $\left[\eta_{2},+\infty\right) \subset\left[\eta_{0},+\infty\right)$.

So, the functions $g_{i}\left(\lambda_{i}, \eta\right)$ for all $\eta \in\left[\eta_{i},+\infty\right)(i=1,2)$ satisfies one of the inequalities

$$
g_{i}\left(\lambda_{i}, \eta\right)>0 \text { or } g_{i}\left(\lambda_{i}, \eta\right)<0 \quad(i=1,2)
$$

Suppose now that for the functions $v_{i}(\eta)(i=1,2)$ limit at $\eta \rightarrow+\infty$ does not exist. Consider the case when one of the 
inequalities (20) is satisfied. As $v_{i}(\eta) \quad(i=1,2)$ are oscillating functions around $\overline{\mathrm{v}}_{\mathrm{i}}=\lambda_{\mathrm{i}} \quad(\mathrm{i}=1,2)$ its graph intersects this straight line infinitely many times in $\left[\eta_{i},+\infty\right), \quad(i=1,2)$. But this is impossible, since in the interval $\left[\eta_{i},+\infty\right),(i=1,2)$ just one of the inequalities (20) are valid and therefore, from (19) it follows that graph of the function $v_{i}(\eta) \quad(i=1,2)$ intersects the straight line $\bar{v}_{i}=\lambda_{i}$ $(i=1,2)$ only once in the interval $\left[\eta_{i},+\infty\right), \quad(i=1,2)$. Accordingly, the function $v_{i}(\eta)(i=1,2)$ has a limit at $\eta \rightarrow+\infty$.

By assumption the functions $v_{i}(\eta)(i=1,2)$ defined in (17), has a limit at $\eta \rightarrow+\infty$. Then $y_{i}^{\prime}(\eta) \quad(i=1,2)$ has a limit at $\eta \rightarrow+\infty$, and this limit is zero. Then

$$
\begin{gathered}
\mathrm{v}_{\mathrm{i}}(\eta)=\left|\frac{d y_{i}}{\mathrm{~d} \eta}+\mathrm{a}_{\mathrm{i} 0}(\eta) \mathrm{y}_{\mathrm{i}}\right|^{\mathrm{p}-2}\left(\frac{\mathrm{dy}}{\mathrm{d} \eta}+\mathrm{a}_{\mathrm{i} 0}(\eta) \mathrm{y}_{\mathrm{i}}\right)= \\
=\left|\mathrm{a}_{\mathrm{i} 0}^{0} \mathrm{y}_{\mathrm{i}}^{0}\right|^{\mathrm{p}-2} \mathrm{a}_{\mathrm{i} 0}^{0} \mathrm{y}_{\mathrm{i}}^{0}+\mathrm{o}(1), \mathrm{i}=1,2 \text { at } \eta \rightarrow+\infty \text {. And by }
\end{gathered}
$$

(18) derivatives of functions $v_{i}(\eta)(i=1,2)$ has a limit at $\eta \rightarrow+\infty$, what are obviously equal to zero.

Consequently, it is necessary

$$
\begin{aligned}
& \lim _{\eta \rightarrow+\infty}\left(a_{i 1}(\eta) v_{i}(\eta)-a_{i 2}(\eta) v_{i}^{\frac{1}{p-1}}(\eta) y_{3-i}-m_{i}\right)+ \\
& +\lim _{\eta \rightarrow+\infty}\left(a_{i 3}(\eta) y_{i} y_{3-i}-m_{i}\right)=0, i=1,2 .
\end{aligned}
$$

From here it can be easily verified that the $\lim _{\eta \rightarrow+\infty} a_{i 1}(\eta)=(p-1)\left(1-\gamma_{i}\right)$,

$\lim _{\eta \rightarrow+\infty} a_{i 2}(\eta)=\frac{(p-1)^{p-1}}{p^{p}}, \quad \lim _{\eta \rightarrow+\infty} a_{i 3}(\eta)=0,(i=1,2)$ if $1-\alpha_{1}(p-2)-\alpha_{2} m_{1} \neq 0$ and $y_{i}(\eta)=c_{i}+o(1) \quad$ as $\eta \rightarrow+\infty$, where $c_{i}(i=1,2)$ are solution of the system of nonlinear algebraic equations $c_{i}^{p-2} c_{3-i} m_{i}=b_{1 i}, i=1,2$ and when $1-\alpha_{1}(p-2)-\alpha_{2} m_{1}=0$ the coefficients $c_{i}, i=1,2$ are solution of the systems of nonlinear algebraic equations

$$
c_{i}^{p-2} c_{3-i}^{m_{i}}=b_{2 i}, \quad i=1,2 .
$$

Consequently, according transformations (15), the compactly supported solution of the problem (8), (9), (11) has an asymptotic of the form (14) at $\xi_{\xi \rightarrow a} \frac{p-1}{p}$.

The theorems are proved.
Corollary 1. A weak solution of the problem (1)-(2) has the following asymptotics

$$
\begin{aligned}
& u_{A}(t, x)=c_{1}(T+t)^{-\alpha_{1}}\left(a-\frac{x^{\frac{p}{p-1}}}{\frac{1}{{ }_{\tau}^{p-1}}}\right)^{\gamma_{1}}(1+o(1)), \\
& \mathrm{v}_{A}(\mathrm{t}, \mathrm{x})=\mathrm{c}_{2}(\mathrm{~T}+\mathrm{t})^{-\alpha_{2}}\left(\mathrm{a}-\frac{\mathrm{x}^{\frac{\mathrm{p}}{\mathrm{p}-1}}}{\frac{1}{\mathrm{p}-1}}\right)^{\gamma_{2}}(1+\mathrm{o}(1)),
\end{aligned}
$$

at ${ }_{x \rightarrow a} \frac{p-1}{p} \frac{1}{p}$, where constants $c_{1}, c_{2}, p, \gamma_{1}, \gamma_{2}, \alpha_{1}, \alpha_{2}$ defined above.

Corollary 2. A weak solution of the problem (1)-(2) has the following asymptotics

$$
\begin{aligned}
& \mathrm{u}_{\mathrm{A}}(\mathrm{t}, \mathrm{x})=\mathrm{c}_{1}(\mathrm{~T}+\mathrm{t})^{-\alpha_{1}}\left(\mathrm{a}-(\mathrm{c} \ln (\mathrm{T}+\mathrm{t})-\mathrm{x}) \frac{\mathrm{p}}{\mathrm{p}-1}\right)^{\gamma_{1}} \times \\
& \times(1+o(1)), \\
& v_{A}(t, x)=c_{2}(T+t)-\alpha_{2}\left(a-(c \ln (T+t)-x) \frac{p}{p-1}\right)^{\gamma_{2}} \times \\
& \times(1+o(1)),
\end{aligned}
$$

at $x \rightarrow c \ln (T+t)-a^{\frac{p-1}{p}}$, where constants $c_{1}, c_{2}, p$ $\gamma_{1}, \gamma_{2}, \alpha_{1}, \alpha_{2}$ defined above.

\section{Fast Diffusion (Case $1<\mathrm{p}<2-\mathrm{m}$,} $\left.\mathrm{m}=\max \left\{\mathrm{m}_{1}, \mathrm{~m}_{2}\right\}\right)$

Suppose that the systems (8), (9) are satisfies the condition

$$
f^{\prime}(0)=0, \phi^{\prime}(0)=0, f(\infty)=0, \phi(\infty)=0 .
$$

Note that the functions $\overline{\mathrm{f}}(\xi)=\left(\mathrm{a}+\xi^{\gamma}\right)^{\gamma_{1}}$, $\bar{\phi}(\xi)=\left(a+\xi^{\gamma}\right)^{\gamma 2}$ satisfies the condition of (22), where $\mathrm{a}>0, \gamma=\frac{\mathrm{p}}{\mathrm{p}-1}, \gamma_{\mathrm{i}}=\frac{(\mathrm{p}-1)\left(\mathrm{p}-2-\mathrm{m}_{\mathrm{i}}\right)}{(\mathrm{p}-2)^{2}-\mathrm{m}_{1} \mathrm{~m}_{2}}, \mathrm{i}=1,2$.

Theorem 4. Let $1<\mathrm{p}<2-\mathrm{m}, \mathrm{m}=\max \left\{\mathrm{m}_{1}, \mathrm{~m}_{2}\right\}$, 


$$
\begin{gathered}
-\frac{1}{p}+b_{i} \geq 0, \quad \gamma\left(\gamma_{i}-m_{i} \gamma_{3}-i\right)\left|\gamma \gamma_{i}\right|^{p-2}=\frac{1}{p}, i=1,2 . \\
u_{0}(x) \geq u_{-}(0, x), v_{0}(x) \geq v_{-}(0, x), x \in R .
\end{gathered}
$$

Then the classical solution $(\mathrm{u}, \mathrm{v})$ of the problem (1)-(2) exists globally and it satisfies estimation

$$
\mathrm{u}(\mathrm{t}, \mathrm{x}) \geq \mathrm{u}_{-}(\mathrm{t}, \mathrm{x}), \mathrm{v}(\mathrm{t}, \mathrm{x}) \geq \mathrm{v}_{-}(\mathrm{t}, \mathrm{x}), \mathrm{x} \in \mathrm{R},
$$

where

$$
\begin{aligned}
& \mathrm{u}_{-}(\mathrm{t}, \mathrm{x})=(\mathrm{T}+\mathrm{t})^{-\alpha_{1}} \overline{\mathrm{f}}(\xi), \\
& \mathrm{v}_{-}(\mathrm{t}, \mathrm{x})=(\mathrm{T}+\mathrm{t})^{-\alpha_{2}} \bar{\phi}(\xi) .
\end{aligned}
$$

The proof of the Theorem 4 is similar to the proof of the Theorem 1.

We introduce the notations

$$
b_{i 3}=\left(1-\frac{1}{p}\right)^{p}\left(\frac{\gamma_{i}}{1-p}-b_{i}\right), b_{i 4}=\left|\gamma_{i}\right|^{1-p}\left(\frac{\gamma_{i}}{p-1}+\frac{1}{p}\right)^{-1},
$$

Theorem 5. Let $1-\alpha_{1}(p-2)-\alpha_{2} m_{1}>0 . \quad$ Then vanishing at infinity solution of the problem (8), (22) has an asymptotic

$$
f(\xi)=c_{3}\left(a+\xi^{\frac{p}{p-1}}\right)^{\gamma_{1}}, \phi(\xi)=c_{4}\left(a+\xi^{\frac{p}{p-1}}\right)^{\gamma_{2}},
$$

at $\xi \rightarrow \infty$, where the coefficients $c_{i}(i=3,4)$ are solution of systems of the nonlinear algebraic equations

$$
c_{3}^{p-2} c_{4}^{m_{1}}=b_{13} b_{14}, c_{4}^{p-2} c_{3}^{m_{2}}=b_{23} b_{24} .
$$

The proof of the Theorem 5 is similar to the proof of the Theorem 2.

Corollary 3. A weak solution of the problem (1)-(2) has an asymptotic the following form

$$
\begin{aligned}
& u_{A}(t, x)=c_{1}(T+t)^{-\alpha_{1}}\left(a+\left(x \tau^{-1 / p}\right) \frac{p}{p-1}\right)^{\gamma_{1}} \times \\
& \times(1+\mathrm{o}(1)), \\
& v_{A}(t, x)=c_{2}(T+t)^{-\alpha_{2}}\left(a+\left(x \tau^{-1 / p}\right) \frac{p}{p-1}\right)^{\gamma_{2}} \times \\
& \times(1+\mathrm{o}(1)),
\end{aligned}
$$

at $\mathrm{x} \tau^{-1 / \mathrm{p}} \rightarrow+\infty$, where constants $\mathrm{c}_{1}, \mathrm{c}_{2}, \mathrm{p}, \gamma_{1}, \gamma_{2}, \alpha_{1}, \alpha_{2}$ defined above.

\section{The Numerical Scheme}

In the domain

$\bar{Q}=\{(t, x): 0 \leq t \leq T, 0 \leq x \leq b\} \quad$ we construct a uniform grid $\bar{\omega}_{\mathrm{h}}$ for $\mathrm{x}$ in steps of $\mathbf{h}$ :

$$
\bar{\omega}_{\mathrm{h}}=\left\{\mathrm{x}_{\mathrm{i}}=\mathrm{ih}, \mathrm{h}>0, \mathrm{i}=0,1, \ldots, \mathrm{n}, \mathrm{hn}=\mathrm{b}\right\},
$$

and a temporary grid

$$
\bar{\omega}_{\kappa}=\left\{t_{j}=j \kappa, \kappa>0, j=0,1, . ., m, \kappa m=T\right\}, T>0 .
$$

Let us replace problem (1) - (2), by using the balance method, implicit difference scheme [22-24] and obtain the difference problem with an error $\mathrm{O}\left(\mathrm{h}^{2}+\kappa\right)$ :

$$
\left\{\begin{array}{l}
\frac{y_{i}^{j+1}-y_{i}^{j}}{\kappa}=c_{i}\left(z^{j}\right) \frac{1}{h^{2}}\left[a_{i+1}\left(y^{j+1}\right)\left(y_{i+1}^{j+1}-y_{i}^{j+1}\right)\right]- \\
-c_{i}\left(z^{j}\right) \frac{1}{h^{2}} a_{i}\left(y^{j+1}\right)\left(y_{i}^{j+1}-y_{i-1}^{j+1}\right), \\
\frac{z_{i}^{j+1}-z_{i}^{j}}{\kappa}=c_{i}\left(y^{j}\right) \frac{1}{h^{2}}\left[a_{i+1}\left(z^{j+1}\right)\left(z_{i+1}^{j+1}-z_{i}^{j+1}\right)\right]- \\
-c_{i}\left(y^{j}\right) \frac{1}{h^{2}} a_{i}\left(z^{j+1}\right)\left(z_{i}^{j+1}-z_{i-1}^{j+1}\right), \\
i=1,2, \ldots, n-1 ; \quad j=0,1, \ldots, m-1, \\
y_{i}^{0}=u_{0}\left(x_{i}\right), z_{i}^{0}=v_{0}\left(x_{i}\right), \quad i=0,1, \ldots, n, \\
y_{0}^{j}=\varphi_{11}\left(t_{j}\right), z_{0}^{j}=\varphi_{21}\left(t_{j}\right), \quad j=1,2, \ldots, m, \\
y_{n}^{j}=\varphi_{12}\left(t_{j}\right), \quad z_{n}^{j}=\varphi_{22}\left(t_{j}\right), \quad j=1,2, \ldots, m,
\end{array}\right.
$$

For calculating $\mathrm{a}(\mathrm{y}), \mathrm{a}(\mathrm{z}), \mathrm{c}(\mathrm{y}), \mathrm{c}(\mathrm{z}), \quad \varphi(\mathrm{t})$ following formulas are used

$$
\begin{gathered}
c_{i}(y)=\left(y_{i}^{j+1}\right)^{m_{2}}, c_{i}(z)=\left(z_{i}^{j+1}\right)^{m_{1}}, \\
a_{i}(y)=\left|\frac{y_{i}^{j+1}-y_{i-1}^{j+1}}{2}\right|^{p-2}, a_{i}(z)=\left|\frac{z_{i}^{j+1}-z_{i-1}^{j+1}}{2}\right|^{p-2},
\end{gathered}
$$




$$
\begin{aligned}
& \varphi_{11}\left(\mathrm{t}_{\mathrm{j}}\right)=\mathrm{c}_{1} \mathrm{a}^{\gamma_{1}}\left(\mathrm{~T}+\mathrm{t}_{\mathrm{j}}\right)^{-\alpha_{1}}, \\
& \varphi_{21}\left(\mathrm{t}_{\mathrm{j}}\right)=\mathrm{c}_{2} \mathrm{a}^{\gamma_{2}}\left(\mathrm{~T}+\mathrm{t}_{\mathrm{j}}\right)^{-\alpha_{2}} \text {, } \\
& \varphi_{12}\left(\mathrm{t}_{\mathrm{j}}\right)=\mathrm{c}_{1}\left(\mathrm{~T}+\mathrm{t}_{\mathrm{j}}\right)^{-\alpha_{1}}\left(\mathrm{a}-\left(\mathrm{b} \tau^{-1 / p}\right) \frac{\mathrm{p}}{\mathrm{p}-1}\right)^{\gamma_{1}}, \\
& \varphi_{22}\left(\mathrm{t}_{\mathrm{j}}\right)=\mathrm{c}_{2}\left(\mathrm{~T}+\mathrm{t}_{\mathrm{j}}\right)^{-\alpha_{2}}\left(\mathrm{a}-\left(\mathrm{b} \tau^{-1 / \mathrm{p}}\right) \frac{\mathrm{p}}{\mathrm{p}-1}\right)^{\gamma_{2}} . \\
& \gamma_{i}=\frac{(p-1)\left(p-2-m_{i}\right)}{(p-2)^{2}-m_{1} m_{2}}, i=1,2 .
\end{aligned}
$$

The system of algebraic equations (24) is nonlinear relative to $\mathrm{y}^{\mathrm{j}+1}, \mathrm{z}^{\mathrm{j}+1}$.

To solve the system of nonlinear equations apply iterative methods and obtained:

$$
\begin{aligned}
& \frac{y_{i}^{j+1}-y_{i}^{j}}{\kappa}=c_{i}\left(z_{i}^{j+1}\right) \frac{1}{h^{2}} a_{i+1}\left(y_{i}^{r} j+1\right)\left(\begin{array}{c}
r+1 \\
y_{i+1}^{j+1}-y_{i}^{j+1}
\end{array}\right)- \\
& -c_{i}\left(\begin{array}{l}
r \\
z_{i}^{j+1}
\end{array}\right) \frac{1}{h^{2}} a_{i}\left(\begin{array}{l}
r \\
y_{i}^{j+1}
\end{array}\right)\left(\begin{array}{ll}
r+1 & r+1 \\
y_{i}^{j+1} & -y_{i-1}^{j+1}
\end{array}\right), \\
& \left\{\frac{z_{i}^{j+1}-z_{i}^{j}}{\kappa}=c_{i}\left(y_{i}^{j} j\right) \frac{1}{h^{2}} a_{i+1}\left(z_{i}^{j+1}\right)\left(\begin{array}{cc}
r+1 & r+1 \\
z_{i+1}^{j+1} & -z_{i}^{j+1}
\end{array}\right)-\right. \\
& \begin{array}{l}
-c_{i}\left(y_{i}^{j+1}\right) \frac{1}{h^{2}} a_{i}\left(z_{i}^{j+1}\right)\left(\begin{array}{lr}
r+1 & r+1 \\
z_{i}^{j+1} & -z_{i-1}^{j+1}
\end{array}\right), \\
i=1,2, \ldots, n-1 ; \\
j=0,1, \ldots, m-1,
\end{array}
\end{aligned}
$$

where $r=0,1,2, \ldots$.

Relatively $\begin{gathered}\mathrm{r}+1 \\ \mathrm{y}_{\mathbf{i}}, \quad \mathrm{z}_{\mathbf{i}}\end{gathered}$ the difference scheme (25) is linear. As an initial iteration for $\begin{array}{cc}r+1 & \mathbf{r}+1 \\ \mathbf{y}_{i}, & \mathbf{z}\end{array}$ is taken from the previous time step: ${ }_{y}^{0}{ }^{j+1}=y^{j},{ }^{j}{ }^{j+1}=z^{j}$. With the score on the iterative scheme are set accuracy of iteration and process continues for as long while the condition $\max _{0 \leq \mathrm{i} \leq \mathrm{n}}\left|\begin{array}{cc}\mathrm{r}+1 & \mathrm{r} \\ \mathrm{y}_{\mathrm{i}}-\mathrm{y}_{\mathrm{i}}\end{array}\right|<\varepsilon, \max _{0 \leq \mathrm{i} \leq \mathrm{n}}\left|\begin{array}{cc}\mathrm{r}+1 & \mathrm{r} \\ \mathrm{z} & \mathrm{i}-\mathrm{z}_{\mathrm{i}}\end{array}\right|<\varepsilon \quad$ is not satisfied.

In (25) we introduce the notation

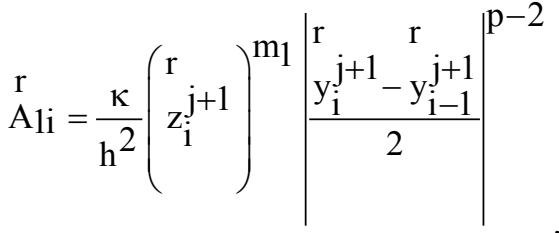

$$
\begin{aligned}
& \left.\stackrel{r}{A} 2 i=\frac{\kappa}{h^{2}}\left(y_{i}^{j}\right)^{j+1}\right)^{m_{2}}\left|\frac{{ }_{i}^{j+1}-z_{i-1}^{j+1}}{2}\right|^{p-2} \\
& \left.\stackrel{r}{B} 1 i=\frac{\kappa}{h^{2}}\left(z_{i}^{r}\right)^{j+1}\right)^{m_{1}}\left|\frac{y_{i+1}^{r}-y_{i}^{j+1}}{2}\right|^{p-2} \\
& \stackrel{r}{B} 2 i=\frac{\kappa}{h^{2}}\left(y_{i}^{r}{ }^{j+1}\right)^{m_{2}}\left|\frac{r}{z_{i+1}^{j+1}-z_{i}^{j+1}}\right|^{p-2}
\end{aligned}
$$

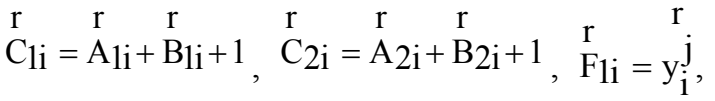

$$
\begin{aligned}
& { }^{r}{ }_{2 i}=z_{i}^{j}, \quad i=1,2, \ldots, n, r=0,1,2, \ldots .
\end{aligned}
$$

Now the difference system (25) can be written as

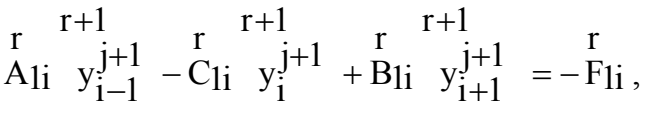

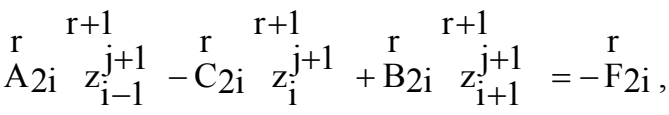

$$
\begin{aligned}
& \mathrm{i}=1,2, \ldots, \mathrm{n}-1 \text {. }
\end{aligned}
$$

For the numerical solution of equations (26) is used tridiagonal matrix algorithm (Thomas algorithm) [22-24]. From the boundary conditions (24), we have

$$
\begin{aligned}
& y_{0}^{j+1}=\varphi_{11}\left(t_{j}+1\right), \quad y_{n}^{j+1}=\varphi_{12}\left(t_{j+1}\right), \\
& z_{0}^{j+1}=\varphi_{21}\left(t_{j}+1\right), \quad z_{n}^{j+1}=\varphi_{22}\left(t_{j+1}\right) .
\end{aligned}
$$

Let

$$
y_{i}^{j+1}=\alpha_{1 i}\left(\beta_{1 i}+y_{i+1}^{j+1}\right), z_{i}^{j+1}=\alpha_{2 i}\left(\beta_{2 i}+z_{i+1}^{j+1}\right),
$$

where

$$
\alpha_{1 i}=\frac{A_{1 i}}{C_{1 i}-\alpha_{1, i-1} B_{1 i}}, \alpha_{2 i}=\frac{A_{2 i}}{C_{2 i}-\alpha_{2, i-1} B_{2 i}},
$$




$$
\begin{gathered}
\beta_{1 i}=\frac{B_{1 i} \alpha_{1, i-1} \beta_{1, i-1}+F_{1 i}}{A_{1 i}}, \\
\beta_{2 i}=\frac{B_{2 i} \alpha_{2, i-1} \beta_{2, i-1}+F_{2 i}}{A_{2 i}}, \\
i=2, \ldots, n
\end{gathered}
$$

The values $\alpha_{11}, \alpha_{21}, \beta_{11}, \beta_{21}$ can be found from (27) and (26) at $i=1$ :

$$
\left\{\begin{array}{l}
\alpha_{11}=\frac{A_{11}}{C_{11}}, \beta_{11}=\frac{B_{11} \phi_{11}+F_{11}}{A_{11}}, \\
\alpha_{21}=\frac{A_{21}}{C_{21}}, \beta_{21}=\frac{B_{21} \phi_{21}+F_{21}}{A_{21}} .
\end{array}\right.
$$

The solution of system (24) is determined by on the recursive formula (27). The values $y_{\mathbf{n}}^{j+1}, z_{\mathbf{n}}^{j+1}$ we obtain from the boundary condition (24)

$$
\left\{\begin{array}{l}
y_{n}^{j+1}=\phi_{12}, z_{n}^{j+1}=\phi_{22} \\
y_{n-1}^{j+1}=\alpha_{1, n-1}\left(\beta_{1, n-1}+y_{n}^{j+1}\right), \\
z_{n-1}^{j+1}=\alpha_{2, n-1}\left(\beta_{2, n-1}+z_{n}^{j+1}\right), \\
\vdots \\
y_{i}^{j+1}=\alpha_{1 i}\left(\beta_{1 i}+y_{i+1}^{j+1}\right) \\
z_{i}^{j+1}=\alpha_{2 i}\left(\beta_{2 i}+z_{i+1}^{j+1}\right) \\
\vdots
\end{array}\right.
$$

To apply the tridiagonal matrix algorithm sufficient to require that the coefficients (26) satisfy the conditions

$$
\left\{\begin{array}{l}
\mathrm{A}_{1 \mathrm{i}} \neq 0, \mathrm{~B}_{1 \mathrm{i}} \neq 0,\left|\mathrm{C}_{1 \mathrm{i}}\right| \geq\left|\mathrm{A}_{1 \mathrm{i}}\right|+\left|\mathrm{B}_{1 \mathrm{i}}\right| \\
\mathrm{A}_{2 \mathrm{i}} \neq 0, \mathrm{~B}_{2 \mathrm{i}} \neq 0, \quad\left|\mathrm{C}_{2 \mathrm{i}}\right| \geq\left|\mathrm{A}_{2 \mathrm{i}}\right|+\left|\mathrm{B}_{2 \mathrm{i}}\right| \quad \mathrm{i}=\overline{1, \mathrm{n}} .
\end{array}\right.
$$

For the iterative scheme (26) tridiagonal matrix algorithm is stable and gives the unique solution.

\section{Numerical Analysis of Solutions}

It is important to choose a proper initial approximation that preserves its nonlinearity properties. On the basis of the above qualitative studies, we produced numerical calculations.

Since we have an asymptotic solution (21) and (23), we can use the numerical data of the asymptotic solution for the initial approximation.
The numerical results show quick convergence of the iterative process to the solution of the Cauchy problem (1)-(2), due to the successful choice of the initial approximation. Below, some numerical experiment results for different numerical parameter values are presented.

Programs for the numerical solution of nonlinear systems not in divergence form developed in MATLAB. Programs are compact. By the user is entered the necessary numerical parameters. At the end of the file automatically displays the calculated results in the form of matrices and graphic. In the same place by running animation can trace the evolution of the process in time.

Let us give some results of numerical experiments. Grid step is small enough $h=0.05$, the number of nodes $N=1000$ and the iteration accuracy is defined $\varepsilon=10^{-3}$. The score was conducted until $t=2$ with a step $\kappa=0.02$.

The initial approximation was taken in the form:

$$
\mathrm{u}_{0}(\mathrm{x})=\overline{\mathrm{u}}(0) \mathrm{f}(\xi), \mathrm{v}_{0}(\mathrm{x})=\overline{\mathrm{v}}(0) \phi(\xi),
$$

where $f(\xi)=c_{1}\left(a-\xi^{\frac{p}{p-1}}\right)^{\gamma_{1}}, \phi(\xi)=c_{2}\left(a-\xi^{\frac{p}{p-1}}\right)^{\gamma_{2}}$,

$$
\begin{gathered}
\overline{\mathrm{u}}(0)=\mathrm{T}^{-\alpha_{1}}, \overline{\mathrm{v}}(0)=\mathrm{T}^{-\alpha_{2}}, \quad \xi=\mathrm{x}[\tau(0)]^{-\frac{1}{\mathrm{p}}}, \\
\tau(0)=\frac{\mathrm{T}^{1-\alpha_{1}(\mathrm{p}-2)-\alpha_{2} \mathrm{~m}_{1}}}{1-\alpha_{1}(\mathrm{p}-2)-\alpha_{2} \mathrm{~m}_{1}}, \gamma_{\mathrm{i}}=\frac{(\mathrm{p}-1)\left(\mathrm{p}-2-\mathrm{m}_{\mathrm{i}}\right)}{(\mathrm{p}-2)^{2}-\mathrm{m}_{1} \mathrm{~m}_{2}}, \mathrm{i}=1,2 .
\end{gathered}
$$

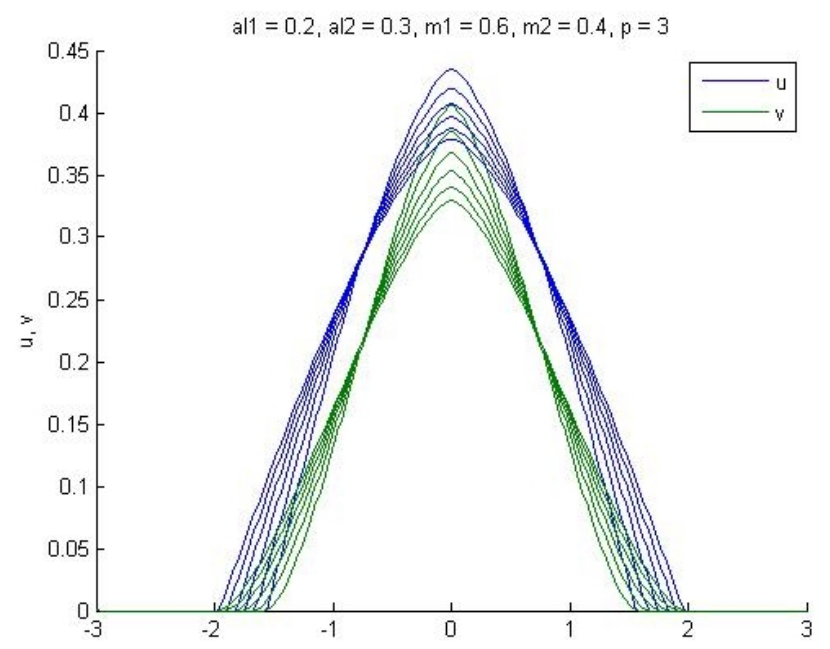

Figure 1. Slow diffusion. Case $\alpha_{1}<1, \alpha_{2}<1, p>2$. 


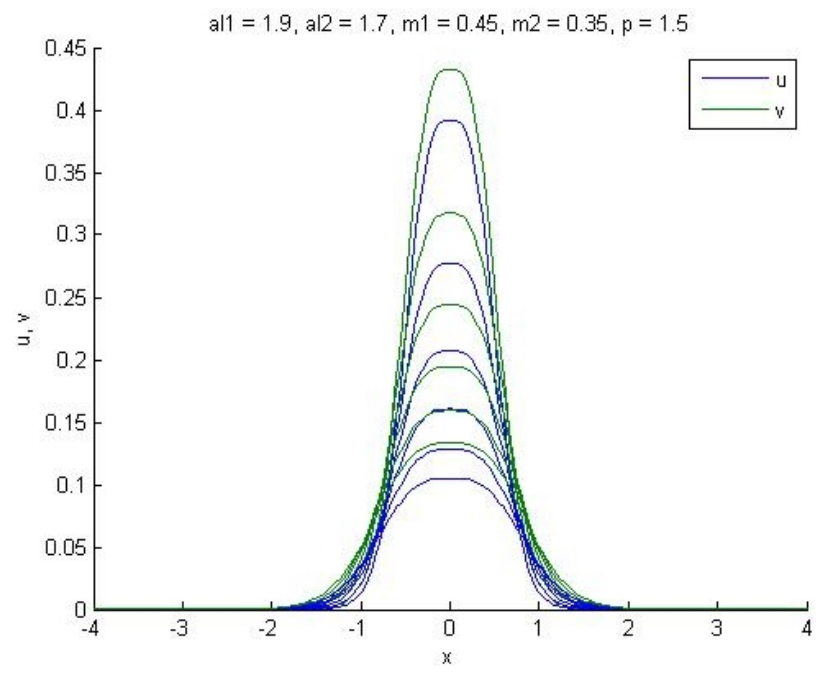

Figure 2. Fast diffusion. Case $\alpha_{1}>1, \alpha_{2}>1, \mathrm{p}<2$.

Results of the numerical experiments and graphs show that the self-similar solutions are very appropriate approximations. Fig.1 shows a compactly supported solution of the problem (1)-(2). In Fig.2 show the properties of the solution of the problem (1)-(2), vanishing at infinity.

Number of iterations is not exceeding of 6 for different values of numerical parameters.

\section{Conclusions}

The property a FSPD of the Cauchy problem for a cross diffusion parabolic system not in divergence form based on comparison method is considered. An asymptotic behavior of self-similar solutions for slow and fast diffusion cases are established. It is shown that coefficients of main terms of asymptotic of solution satisfy to some system of a nonlinear algebraic equation.

Results of computational experiments show that the self-similar solutions are very appropriate approximation. And the iterative method based on the Picard's method is effective for the solution of nonlinear problems and retains the nonlinear effects, if we will use as an initial approximation the solutions of self-similar equations, constructed by the method of nonlinear splitting and by the method of standard equation [14-21].

\section{REFERENCES}

[1] A. Friedman, J.B. McLeod. Blow up of solutions of nonlinear degenerate parabolic equations. Archive for Rational Mechanics and Analysis, 96 (1), 55-80, 1986.

[2] M.E. Gage. On the size of the blow-up set for a quasilinear parabolic equation. Contemp. Math., 127, 41-58, 1992.

[3] R.D. Passo, S. Luckhaus. A degenerate diffusion problem not in divergence form. J. differential equations, 69, 1, 1-14, 1987.

[4] S. Wang, M.X. Wang, C.H. Xie. A nonlinear degenerate diffusion equation not in divergence form, Z. Angew. Math. Phys., 51, 149-159, 2000.

[5] T. Zimmer. On a degenerate parabolic equation. IWR Heidelberg, Preprint, 93-05, 1993.

[6] H.W. Chen. Analysis of Blowup for a Nonlinear Degenerate Parabolic Equation. Journal of Mathematical Analysis and Applications, Volume 192, Issue 1, 180-193, 1995.

[7] M. Wiegner. Blowup for solutions of some degenerate parabolic equations. Differential and Integral Equations, Volume 7, Number 6, 1641-1647, 1994.

[8] J.R. Raimbekov. The Properties of the Solutions for Cauchy Problem of Nonlinear Parabolic Equations in Non-Divergent Form with Density. Journal of Siberian Federal University. Mathematics and Physics, 8(2), 192-200, 2015.

[9] S. Zhou, X. Tang, C. Yang. A doubly degenerate diffusion equation not in divergence form with gradient term. Boundary Value Problems, Vol. 2016, 126, 1-19, 2016.

[10] M.X. Wang, C.H. Xie. A degenerate strongly coupled quasilinear parabolic system not in divergence form. Z. Angew. Math. Phys., 55, 741-755, 2004.

[11] J. Wang. Behaviors of solutions to a class of nonlinear degenerate parabolic equations not in divergence form. Applied Mathematics Letters, Volume 24, Issue 2, 191-195, 2011.

[12] Haihua Lu. Global existence and blow-up analysis for some degenerate and quasilinear parabolic systems. Electronic Journal of Qualitative Theory of Differential Equations, 49, $1-14,2009$.

[13] A.A. Samarskii, V.A. Galaktionov, S.P. Kurdyumov and A.P. Mikhailov. Blow-Up in Quasilinear Parabolic Equations, Berlin, 4, Walter de Grueter, 535, 1995.

[14] M. Aripov. Method of the Standard Equation for the Solution of the Nonlinear Value Problem, Fan, Tashkent, 137, 1988.

[15] M. Aripov, Sh.A. Sadullaeva. Qualitative Properties of Solutions of a Doubly Nonlinear Reaction Diffusion System with a Source. Journal of Applied Mathematics and Physics, 3 , 1090-1099, 2015.

[16] M. Aripov, Sh.A. Sadullaeva. An asymptotic analysis of a self-similar solution for the double nonlinear reaction-diffusion system, J. Nanosystems: physics, chemistry, mathematics, 6 (6), 1-10, 2015.

[17] M. Aripov, Z. Rakhmonov. Estimates and Asymptotic of Self-similar Solutions to a Nonlinear Filtration Problem with Variable Density and Nonlocal Boundary Conditions, Universal Journal of Computational Mathematics, 4(1), 1-5, 2016 .

[18] Sh.A. Sadullaeva. Numerical Investigation of Solutions to a Reaction-Diffusion System with Variable Density, Journal of Siberian Federal University. Mathematics and Physics, V. 9 (1), 90-101, 2016.

[19] M. Aripov, A.S. Matyakubov. To the qualitative properties of solution of system equations not in divergence form. International Journal of Innovative Science, Engineering and 
Technology, Vol. 3 Issue 8, 533-537, 2016.

[20] M. Aripov, A.S. Matyakubov. To the properties solutions of a cross-diffusion parabolic system not in divergence form. Universal Journal of Computational Mathematics, 5(1), 1-7, 2017.

[21] M. Aripov, A.S. Matyakubov. Self-similar solutions of a cross-diffusion parabolic system with variable density: explicit estimates and asymptotic behavior. Nanosystems: physics, chemistry, mathematics, 8 (1), 43-50, 2017.
[22] M. El-Mikkawy, F. Atlan. Algorithms for Solving Linear Systems of Equations of Tridiagonal Type via Transformations, Applied Mathematics, 5, 413-422, 2014.

[23] W.F. Ames. Numerical Methods for Partial Differential Equations. Third Edition. Academic Press, Mathematics, 451 pages, 2014.

[24] A.S. Matyakubov. Difference scheme of the second order of accuracy for the nonlinear cross-diffusion system not in divergence form, ACTA NUUz, 2/1, 133-141, 2017. 